\title{
Spontaneous Reduction Finding: Magnetic Resonance Imaging Evaluation of Segmental Instability in Spondylolisthesis
}

\author{
Jae Yoon Chung, Sung Kyu Kim, Sung Taek Jung, Keun Bae Lee, \\ Hyoung Yeon Seo, Chang Yong Hu, Gi Heon Park \\ Department of Orthopedic Surgery, Chonnam National University Hospital, \\ Chonnam National University Medical School, Gwangju, Korea
}

\begin{abstract}
Study Design: Retrospective chart review.
Purpose: To assess whether spontaneous reduction of spondylolisthesis, as seen on magnetic resonance imaging (MRI), is related to the degree of segmental instability and low back pain.

Overview of Literature: The flexion-extension radiographs obtained in the sagittal plane are frequently used when segmental instability of spondylolisthesis is evaluated.

Methods: We retrospectively reviewed 137 patients and measured the differences of the percentage of sagittal translation and sagittal angulation to determine the segmental instability between the flexion and extension radiographs, and the spontaneous reduction on MRI. We then compared the degrees of segmental instability and the degrees of spontaneous reduction. To assess the effect of low back pain on segmental motion in regards to the flexion-extension radiographs, we compared the preoperative visual analogue scales (VAS) score for low back pain between the more and the less spontaneous reduction groups.

Results: The mean degree of spontaneous reduction was 5.2\%. A statistically significant correlation was found between the sagittal translation on the flexion-extension radiographs and the degree of spontaneous reduction $(r=0.557, p<0.001)$ and between the sagittal angulation on the flexion-extension radiographs and the degree of spontaneous reduction $(r=0.215, p$ $=0.012$ ) The preoperative VAS scores for low back pain of the more spontaneous reduction group and the less spontaneous reduction group were 4.6 and 3.6 points, respectively, and this difference was statistically significant $(p=0.002)$.

Conclusions: Spontaneous reduction of spondylolisthesis on MRI was found to be closely related to segmental instability, and the degree of spontaneous reduction seen on MRI could be useful for the evaluation of segmental instability in patients with spondylolisthesis, especially with severe low back pain.
\end{abstract}

Key Words: Spondylolisthesis, Segmental instability, Spontaneous reduction, Magnetic resonance imaging

\section{Introduction}

Spondylolisthesis with segmental instability often causes central or foraminal stenosis, and low back pain $[1,2]$. Clinically, dynamic flexion-extension radiographs are frequently used to identify segmental instability and magnetic resonance imaging (MRI) is usually used to identify central or

\section{foraminal stenosis.}

We have observed the spontaneous reduction of the listhetic segment of patients with segmental instability on MRI (Fig. 1). We suggested that this phenomenon might be due to segmental instability. It has been found that low back pain and paraspinal muscle spasm could inhibit the maximal segmental motion [3-6]. If patients have low back pain

Received Jan 29, 2012; Revised Sep 20, 2012; Accepted Sep 27, 2012

Corresponding author: Sung Kyu Kim, MD

Department of Orthopedics, Chonnam National University Hospital, Chonnam National University Medical School,

42 Jebong-ro, Dong-gu, Gwangju 501-757, Korea

Tel: +82-62-227-1640,Fax:+82-62-225-7794, E-mail: bonjourksk@hanmail.net

Copyright (C) 2012 by Korean Society of Spine Surgery

This is an Open Access article distributed under the terms of the Creative Commons Attribution Non-Commercial License (http://creativecommons.org/licenses/by-nc/3.0/) which permits unrestricted non-commercial use, distribution, and reproduction in any medium, provided the original work is properly cited. Asian Spine Journal • pISSN 1976-1902 eISSN 1976-7846 
and paraspinal muscle spasm, the flexion-extension radiographs may not accurately reflect the degree of segmental instability. The MRI is checked in the supine position and requires more imaging time than the plain radiograph with 20 to 30 minutes. It can relieve pain and paraspinal muscle spasms during imaging, and the relief may reduce anterior slippage in segmental instability. Based on this hypothesis, the purpose of this study was to assess whether spontaneous reduction of spondylolisthesis on the MRI was related to the degree of segmental instability and low back pain.

\section{Materials and Methods}

We retrospectively reviewed the details of 137 patients, who underwent posterior lumbar interbody fusion ( $\mathrm{n}=$ 111) or transforaminal lumbar interbody fusion $(n=26)$ for spondylolisthesis at one level between 2003 and 2009. Every patient had a walking disability because of neurologic claudication or an associated neurologic deficit in the form of muscle weakness or sensory deficits in the distribution of the affected nerve root, preoperatively. We excluded those patients with multi-segment spinal disease and a previous spinal operation; in order to minimize the bias from their ef- fects to the segmental motion of the listhetic segment. The mean age of the patients was 59.6 years (range, 43 to 79 years); 27 were men and 110 were women. Ninety-three patients had degenerative spondylolisthesis and 44 had isthmic spondylolisthesis. The L3-4 segment was involved in 10 patients; L4-L5 in 101, and L5-S1 in 26. One hundred and twelve patients demonstrated grade I (less than $25 \%$ anterior slippage) and 25 patients demonstrated grade II (25-50\% anterior slippage). The neutral anterior-posterior and lateral radiographs, and dynamic flexion-extension radiographs in the lateral decubitus position were checked in all patients. In addition, each patients MRI was studied for preoperative evaluation. Clinically, we reviewed the preoperative Visual analog scale (VAS) score for low back pain on the activity of daily living. To evaluate the degree of segmental instability of the listhetic segments, we measured the differences of the percentage of sagittal translation and the differences of the sagittal angulation between the flexion and extension radiographs. The difference of sagittal translation and sagittal angulation was checked three times by one orthopaedic surgeon. We used the average of the three values. Sagittal translation was measured using the methods described by White and Panjabi [7], and sagittal angulation was defined
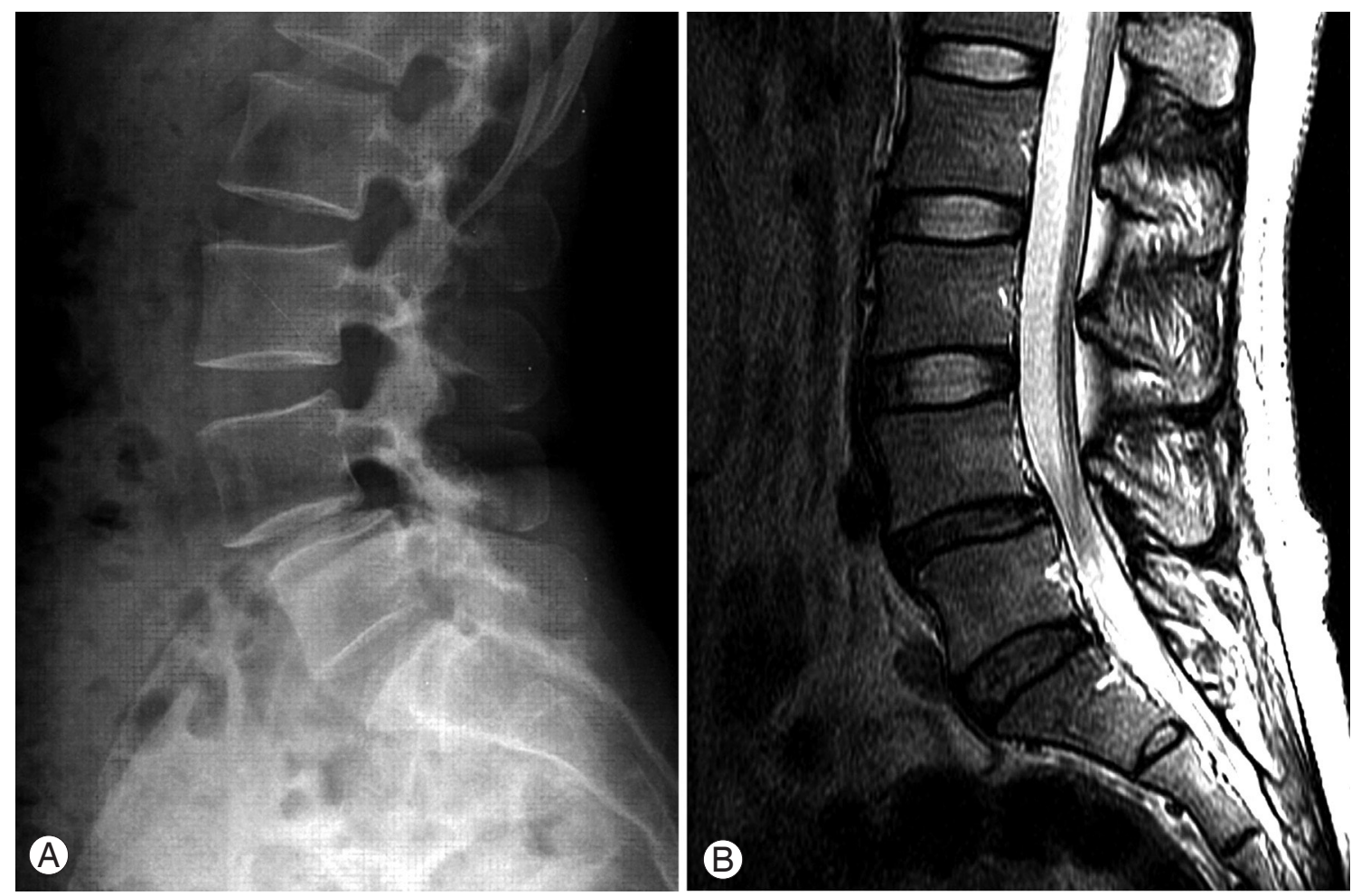

Fig. 1. A 36-year-old woman with L4-5 degenerative spondylolisthesis. (A) The preoperative neutral lateral radiograph demonstrates a $13.6 \%$ anterior translation. (B) On magnetic resonance imaging, the anterior translation is reduced to $2.3 \%$. 
Table 1. Radiographic results of sagittal translation and sagittal angulation $(n=137)$

\begin{tabular}{|c|c|c|c|c|}
\hline & Flexion & Neutral & Extension & $\begin{array}{c}\text { Magnetic resonance } \\
\text { imaging }\end{array}$ \\
\hline Sagittal translation (\%) & $22.1 \pm 7.6$ & $17.7 \pm 6.9^{\mathrm{a})}$ & $14.8 \pm 6.9$ & $12.5 \pm 6.6^{\mathrm{a})}$ \\
\hline Sagittal angulation $\left({ }^{\circ}\right)^{\mathrm{c})}$ & $-2.4 \pm 5.4$ & $3.0 \pm 4.9^{\mathrm{b})}$ & $5.8 \pm 5.2$ & $3.6 \pm 4.7^{\mathrm{b})}$ \\
\hline
\end{tabular}

Values are presented as mean \pm standard deviation.

${ }^{\text {a) }} P<0.001,{ }^{\text {b) }} P=0.026,{ }^{\text {c) }}$ Positive value $=$ lordosis, negative value $=$ kyphosis.

as the angle between the lower end plate of the upper vertebral body and the upper end plate of the lower vertebral body (Fig. 2). The segmental instability was classified according to Wiltse's criteria [8]. Unstable translation and unstable angulation were defined as the differences regarding the percentages of sagittal translations and the degrees of the sagittal angulations between the flexion and extension radiographs in patients with segmental instability. In addition, we measured the spontaneous reduction. For spontaneous reduction, translational spontaneous reduction was defined as the difference in the percentages of sagittal translations and angulational spontaneous reduction was defined as the difference in the degree of sagittal angulations between the neutral lateral radiograph and mid-sagittal section of the MRI in each patient. We then compared the degree of segmental instability with the degree of spontaneous reduc-

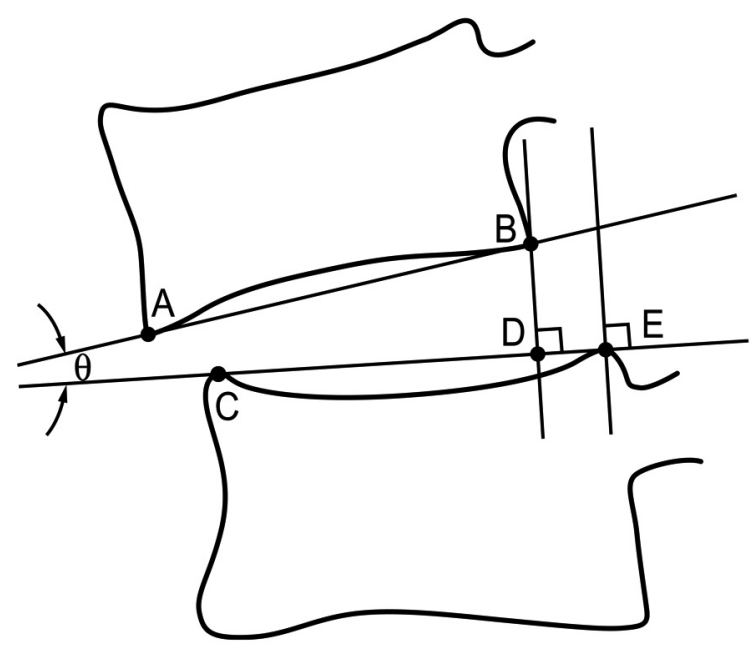

Fig. 2. Measurement of sagittal translation and sagittal angulation. Sagittal translation (\%): The length between $\mathrm{D}$ and E/the length between $\mathrm{C}$ and $\mathrm{E} \times 100$, Sagittal angulation $\left({ }^{\circ}\right)$ : $\theta$ is angle between $\mathrm{AB}$ line and $\mathrm{CE}$ line, $\mathrm{A}$ : Anterior margin of lower endplate of superior vertebra, B: Posterior margin of lower endplate of superior vertebra, $\mathrm{C}$ : Anterior margin of upper endplate of inferior vertebra, D: Crossing point between $\mathrm{CE}$ line and the perpendicular line to $\mathrm{CE}$ line from $\mathrm{B}$ E: Posterior margin of upper endplate of inferior vertebra. tion. To identify the effect of low back pain to the segmental motion on the flexion-extension radiographs, we compared the preoperative VAS score for low back pain between the more and the less spontaneous reduction groups; the more spontaneous reduction group was defined as the spontaneous reduction was more than the difference between the flexion and extension radiographs and the less spontaneous reduction group was defined as the spontaneous reduction was less than-the difference between the flexion and extension radiographs. Above definitions were equally applied to sagittal translation and sagittal angulation.

To identify the factors that have an influence on the spontaneous reduction, the spontaneous reductions were compared according to age, gender, pelvic incidence, lumbar lordosis (between L1 and S1), the type of spondylolisthesis and the listhetic segment level. The correlations between segmental instability and the spontaneous reduction were analyzed using Pearson's correlation coefficient. Other comparisons were analyzed using the paired t-test and the independent t-test. Probability values of $<0.05$ were considered statistically significant.

\section{Results}

The sagittal translations were $22.1 \pm 7.6 \%, 17.7 \pm 6.9 \%$, $14.8 \pm 6.9 \%$, and $12.5 \pm 6.6 \%$ on the flexion, neutral and extension radiographs and MRI, respectively. The differences of the sagittal translations between the neutral lateral radiograph and the mid-sagittal section of MRI were statistically significant $(p<0.001)$. The translational spontaneous reduction was $5.2 \pm 4.0 \%$. The sagittal angulations were $-2.4 \pm 5.4^{\circ}$ lordosis, $3.0 \pm 4.9^{\circ}$ lordosis, $5.8 \pm 5.2^{\circ}$ lordosis and $3.6 \pm 4.7^{\circ}$ lordosis on the flexion, neutral and extension radiographs and MRI, respectively (Table 1). The angulational spontaneous reduction was $-0.6 \pm 4.0^{\circ}$. A statistically significant correlation was found between the unstable translation and the translational spontaneous reduction $(r=0.557, p<0.001)$, and between the unstable angulation and the angulational 
Table 2. The degree of spontaneous reduction according to the age, gender, plvic incidence, lumbar lordosis, the type of spondylolisthesis and the level of the listhetic segment

\begin{tabular}{llcc}
\hline \hline & & Spontaneous reduction $(\%)$ & $p$-value \\
\hline Age $(\mathrm{yr})$ & $<60(\mathrm{n}=65)$ & $6.5 \pm 4.2$ & 0.066 \\
& $\geq 60(\mathrm{n}=72)$ & $4.0 \pm 3.4$ & 0.048 \\
Gender & Male $(\mathrm{n}=27)$ & $4.0 \pm 3.0$ & \\
& Female $(\mathrm{n}=110)$ & $5.5 \pm 4.1$ & 0.073 \\
Pelvic incidence $\left(^{\circ}\right)$ & $<55(\mathrm{n}=64)$ & $4.8 \pm 3.3$ & \\
& $\geq 55(\mathrm{n}=73)$ & $5.6 \pm 4.5$ & 0.333 \\
Lumbar lordorsis $\left(^{\circ}\right)$ & $<40(\mathrm{n}=91)$ & $5.4 \pm 4.1$ & \\
& $\geq 40(\mathrm{n}=46)$ & $4.8 \pm 3.7$ & \\
Type of spondylolisthesis & Degenerative $(\mathrm{n}=93)$ & $4.7 \pm 3.7$ & 0.661 \\
& Isthmic $(\mathrm{n}=44)$ & $6.4 \pm 4.4$ & \\
Level of listhetic segment & L3-4 $(\mathrm{n}=10)$ & $4.3 \pm 3.1$ & \\
& L4-5 $(\mathrm{n}=101)$ & $5.2 \pm 3.9$ & \\
& L5-S1 $(\mathrm{n}=26)$ & $5.2 \pm 4.0$ & \\
\hline
\end{tabular}

Values are presented as mean \pm standard deviation.

spontaneous reduction $(r=0.215, p=0.012)$. These results demonstrated that the spontaneous reduction is closely related to the segmental instability.

The mean preoperative VAS score for low back pain in regards to all patients was 3.8 points. Thirty seven patients were in the more translational spontaneous reduction group. The mean VAS score was $4.6 \pm 1.7$ points, which was significantly more than that of the less translational spontaneous reduction group $(3.6 \pm 1.3$ points $)(p=0.002)$. Thirty seven patients were in the more angulational spontaneous reduction group. The mean VAS score was $6.1 \pm 2.1$ points, which was significantly more than that of the less angulational spontaneous reduction group ( $4.0 \pm 0.8$ points) ( $p=$ $0.001)$.

\section{Discussion}

Although the value of flexion-extension radiographs is controversial, the lateral flexion-extension radiographs are most widely used by clinicians to assess segmental instability. Many reports have emphasized the importance of various postures in checking the radiographs, standing [9-11], lateral decubitus [12], traction-compression [6] and the supine prone position [13]. Several authors have recently reported on the value of continuous motion analysis techniques using video-fluoroscopy to assess lumbar spinal movement [14-
16]. We used flexion-extension radiographs in the lateral decubitus position to assess segmental instability in spondylolisthesis patients because this technique is simple and economic to use in routine clinical practice.

In order to identify whether the spontaneous reduction of spondylolisthesis can be observed on MRI, we compared the sagittal translations and sagittal angulations between the neutral lateral radiographs and the mid-sagittal sections of MRI and we observed a mean $5.2 \%$ of translational spontaneous reduction and $-0.6^{\circ}$ of angulational spontaneous reduction. Passive reduction of spondylolisthesis has been previously observed. Montgomery and Fischgrund [17] found that many low-grade lumbar spondylolisthetic deformities reduced almost completely on the operating table after the administration of an anesthetic and that the percentage of slip decreased $24 \%, 15 \%$, and $6 \%$ on the flexion, extension, and intraoperative lateral radiographs, respectively.

Next, after attempting to correlate segmental instability and spontaneous reduction, we found a statistically significant correlation. The clinical significance of this result was that spontaneous reduction of anterior slippage on the MRI could be a useful indicator of segmental instability. According to our results, the relationship between VAS score and segmental instability showed a statistically significant difference. These results could indicate the relevance be- 
tween instability and the patient condition and also with the amount of the spontaneous reduction rate during the MRI.

We also considered that muscle relaxation might contribute to the spontaneous reduction of anterior slippage of an unstable segment on the MRI. Pearcy et al. [5] reported that patients with low back pain had restricted primary movement at the lower lumbar level, and they suggested that this was due to the splinting action of muscles. Wood et al. [12] reported that the maximal abnormal translatory motion occurred on the flexion-extension radiographs taken in the lateral decubitus position and they explained that this finding was due to splinting from the paraspinal postural musculature or the abdominal musculature, which reduced the allowable motion when the patient was standing and this forced the patients to support themselves against gravity.

In our study, we compared the preoperative VAS score for low back pain between the more and the less spontaneous reduction groups, and the result was that the more spontaneous reduction group had significantly more severe low back pain. This result suggested that, in patients with severe low back pain in the case of spondylolisthesis and segmental instability, the flexion-extension radiographs could not adequately reflect the segmental instability. In this situation, MRI might be more suitable to assess the segmental instability because spontaneous reduction was closely related to segmental instability.

When we evaluated the factors that had an influence on the spontaneous reduction of anterior slippage, women were found to show significantly more spontaneous reduction than men. This result might be due to the fact that women have smaller volumes of paraspinal muscles than men but more study on this is needed (Table 2).

A limitation of the study was that we did not check the degree of facet joint arthritis, the shape of the facet joint, and disc height as reason for segmental instability. Thus, further investigation is needed to evaluate the reason for the spontaneous reduction of anterior slippage.

\section{Conclusions}

On MRI, spondylolisthesis was reduced to some extent and this spontaneous reduction was found to be closely related to segmental instability. Low back pain may reduce segmental motion, and so the flexion-extension radiographs may not accurately reflect the degree of segmental instability. The present study indicates that the degree of spontaneous reduction seen on MRI could be useful for evaluation of segmental instability in patients with spondylolisthesis, especially those with severe low back pain.

\section{REFERENCES}

1. Stokes IA, Frymoyer JW. Segmental motion and instability. Spine (Phila Pa 1976) 1987;12:688-91.

2. Esses SI, Botsford DJ, Kostuik JP. The role of external spinal skeletal fixation in the assessment of low-back disorders. Spine (Phila Pa 1976) 1989;14:594-601.

3. Kirkaldy-Willis WH, Farfan HF. Instability of the lumbar spine. Clin Orthop Relat Res 1982;(165):110-23.

4. Dupuis PR, Yong-Hing K, Cassidy JD, Kirkaldy-Willis WH. Radiologic diagnosis of degenerative lumbar spinal instability. Spine (Phila Pa 1976) 1985;10:262-76.

5. Pearcy M, Portek I, Shepherd J. The effect of low-back pain on lumbar spinal movements measured by threedimensional X-ray analysis. Spine (Phila Pa 1976) 1985;10:150-3.

6. Friberg O. Lumbar instability: a dynamic approach by traction-compression radiography. Spine (Phila $\mathrm{Pa}$ 1976) 1987;12:119-29.

7. White AA 3rd, Panjabi MM. Clinical biomechanics of the spine. 2nd ed. Philadelphia: Lippincott Williams \& Wilkins; 1990.

8. Wiltse LL, Rothman SL. Spondylolisthesis: classification, diagnosis and natural history. Semin Spine Surg 1989;1;78-94.

9. Knutsson F. The instability associated with disk degeneration in the lumbar spine. Acta Radiol 1944;25:593609.

10. Boden SD, Wiesel SW. Lumbosacral segmental motion in normal individuals. Have we been measuring instability properly? Spine (Phila Pa 1976) 1990;15:571-6.

11. Dvorák J, Panjabi MM, Novotny JE, Chang DG, Grob D. Clinical validation of functional flexion-extension roentgenograms of the lumbar spine. Spine (Phila $\mathrm{Pa}$ 1976) 1991;16:943-50.

12. Wood KB, Popp CA, Transfeldt EE, Geissele AE. Radiographic evaluation of instability in spondylolisthesis. Spine (Phila Pa 1976) 1994;19:1697-703.

13. D'Andrea G, Ferrante L, Dinia L, Caroli E, Orlando ER. "Supine-prone" dynamic X-ray examination: new method to evaluate low-grade lumbar spondylolisthesis. J Spinal Disord Tech 2005;18:80-3.

14. Lee SW, Wong KW, Chan MK, Yeung HM, Chiu JL, Leong JC. Development and validation of a new tech- 
nique for assessing lumbar spine motion. Spine (Phila Pa 1976) 2002;27:E215-20.

15. Otani K, Okawa A, Shinomiya K, Nakai O. Spondylolisthesis with postural slip reduction shows different motion patterns with video-fluoroscopic analysis. J Orthop Sci 2005;10:152-9.
16. Teyhen DS, Flynn TW, Childs JD, et al. Fluoroscopic video to identify aberrant lumbar motion. Spine (Phila Pa 1976) 2007;32:E220-9.

17. Montgomery DM, Fischgrund JS. Passive reduction of spondylolisthesis on the operating room table: a prospective study. J Spinal Disord 1994;7:167-72. 